\title{
Metformin Use and Metformin-associated Lactic Acidosis in Intensive Care Unit Patients with Diabetes
}

\author{
Venkat Rajasurya $^{1}$, Humayun Anjum ${ }^{2}$, Salim Surani ${ }^{3}$ \\ 1. Pulmonary Critical Care, Decatur Memorial Hospital, Decatur, USA 2. Pulmonary Critical Care, Corpus \\ Christi Medical Center, Corpus Christi, USA 3. Internal Medicine, Texas A\&M Health Science Center, \\ Temple, USA
}

$\square$ Corresponding author: Venkat Rajasurya, vrajasurya@gmail.com

Disclosures can be found in Additional Information at the end of the article

\section{Abstract}

Metformin is a very potent anti-diabetic drug that has become the drug of choice for the treatment of type 2 diabetes. In addition to its glucose-lowering properties, it also reduces allcause mortality through its anti-inflammatory and cardioprotective effects. Although metformin-associated lactic acidosis (MALA) is a very rare event, the mortality associated with it is close to $50 \%$. As it is excreted through the kidney, MALA is frequently seen in patients on metformin with risk factors for developing acute kidney injury. Metformin increases the plasma lactate level in a concentration-dependent manner by inhibiting mitochondrial respiration, usually in the presence of a secondary event that disrupts lactate production or clearance. The incidence of acute kidney injury is very high in critically ill patients contributed by circulatory defects as well as contrast-induced nephropathy, the incidence of which is also high in this subset of the population. Because of this potential risk, metformin is frequently discontinued in diabetic patients admitted to the intensive care unit. Blood glucose variability and hypoglycemia, however, are both related to poor intensive care unit (ICU) outcomes and in order to prevent this in diabetic patients admitted to ICU, oral hypoglycemic agents are frequently switched to intravenous or subcutaneous insulin regimens, which allows for closer monitoring and better blood glucose control.

Received 04/08/2019

Review began 04/13/2019

Review ended 05/17/2019

Published 05/23/2019

(c) Copyright 2019

Rajasurya et al. This is an open access article distributed under the terms of the Creative Commons Attribution License CC-BY 3.0., which permits unrestricted use, distribution, and reproduction in any medium, provided the original author and source are credited.
Categories: Endocrinology/Diabetes/Metabolism, Internal Medicine

Keywords: metformin associated lactic acidosis, acute kidney injury, diabetes, metformin, icu, sepsis, metformin toxicity, blood glucose, antihyperglycemic, insulin

\section{Introduction And Background}

Metformin and phenformin are derivatives of galegine, which was used in herbal medicine in medieval Europe [1]. The most significant benefit of metformin is reduction in all-cause mortality [2]. But, other benefits include relatively low cost, absence of weight gain, low level of side effects, and anti-inflammatory and antioxidant properties. Hence, it is recommended as the first line treatment [3]. Despite all the benefits, metformin is contraindicated in a certain subgroup of patients. It can cause lactic acidosis, which is rare, but can be potentially fatal [4]. Most commonly, it occurs in patients with a condition that impairs the lactate production or its clearance. Critically ill patients who are on metformin are at a higher risk for this complication as they are more prone to develop acute kidney injury (AKI), hepatic failure, respiratory failure, and circulatory shock in an intensive care unit (ICU) setting. With the increase in the percentage of elderly patients being managed in the ICU, this potential risk further increases. To avoid potential complications and to achieve a better blood glucose control, metformin is 


\section{Cureus}

commonly switched to insulin in ICU patients.

\section{Review}

\section{Metformin metabolism and mechanism of action}

Metformin is a unique anti-diabetic drug, as it does not cause hypoglycemia [2]. This is due to its characteristic pharmacokinetics. Following oral administration of metformin, a major proportion is absorbed and the rest is excreted in the stool. Additionally, almost all of the absorbed drug is eliminated unchanged via kidneys. The major site of action of metformin is the liver [5]. It not only reduces the glucose output by inhibiting the endogenous glucose production but also augments glucose uptake in the peripheral tissues due to improved insulin sensitivity [6].

\section{Metformin and lactic acidosis}

\section{Lactic Acidosis}

Lactate accumulates during hypoxia because of glycolysis and is produced by various organs including but not limited to the gut, liver and peripheral tissues [7]. Figure 1 clearly outlines the biochemistry of lactate production. When there is sufficient oxygen supply, glucose is metabolized to form water and carbon dioxide. This process results in the production of adenosine triphosphate (ATP), which is the energy carrier of the cell. Hypoxic conditions promote increased glycolysis to provide additional ATP. The excess pyruvate produced during this process is converted into lactate and subsequently released into the bloodstream where it accumulates over time [7].

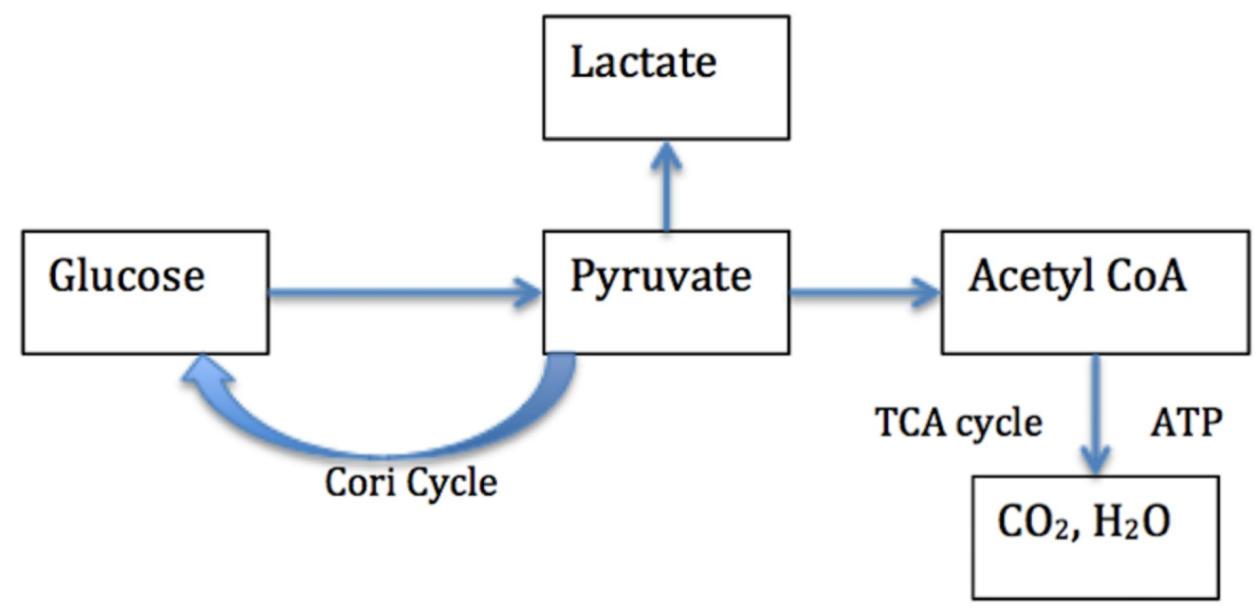

\section{FIGURE 1: Biochemistry of lactate production}

Pyruvate is produced from glucose via glycolysis. (1) Under adequate oxygen availability, pyruvate is oxidized to $\mathrm{CO} 2$ and $\mathrm{H} 2 \mathrm{O}$ in the tricarboxylic acid (TCA) cycle (2) Under anaerobic conditions, pyruvate is unable to enter mitochondria and is reduced to lactate. (3) Pyruvate can be converted to glucose in the liver and kidney by the Cori cycle. Lactate accumulation occurs under anaerobic conditions. 


\section{Cureus}

\section{Metformin-induced Lactic Acidosis}

Metformin has several effects on pathways involving lactate. Metformin inhibits gluconeogenesis by blocking pyruvate carboxylase and this leads to lactate accumulation [8]. It also augments lactate production in insulin-dependent tissue, promotes glycolysis due to mitochondrial impairment and activates anaerobic metabolism of glucose in the intestine. Under normal circumstances, at therapeutic levels of metformin, this has no significant effect on the accumulation of lactate as it is converted back to glucose via the Cori cycle. If there is an accumulation of metformin due to either defective elimination or excess intake, there is a decrease in lactate uptake by the liver and lactic acidosis ensues. Not everyone with an isolated metformin overdose develops lactic acidosis or significant hyperlactatemia [9].

Metformin-associated Lactic Acidosis (MALA)

MALA remains an important adverse event due to its increased mortality. Table 1 highlights the three likely scenarios in which patients treated with metformin can develop lactic acidosis [10].

\section{Mechanism}

Promote the formation of lactate by the peripheral tissues because of hypoxia

Impair lactate metabolism through the pathway of gluconeogenesis

Dramatically increase levels of metformin

\section{Clinical Conditions}

Cardiac failure, Sepsis, Severe dehydration,

Respiratory failure

Primary or secondary hepatic failure

Renal impairment

\section{TABLE 1: Conditions that may increase the risk of lactic acidosis associated with metformin}

Erythrocyte levels and an assay of blood levels of metformin can be helpful to estimate the risk and extent of metformin accumulation. This can be extremely complicated and perhaps not very reliable due to broad range of observed concentrations and continuous distribution of values [11]. Additionally, there is not much data regarding this as well. MALA without an associated condition is exceptional and thus is not necessarily due to metformin accumulation.

\section{Risk Factors for Mala}

It is the imbalance between the production of lactate and reduced clearance of lactate leads to MALA. MALA needs to be considered when the metformin level is more than $5 \mu \mathrm{g} / \mathrm{mL}$ (therapeutic range $<2 \mu \mathrm{g} / \mathrm{mL}$ ) [12]. The common risk factors for MALA are listed in Table 2 [10]. In general, the metformin plasma concentrations are two to four-fold higher in patients with type 2 diabetes and moderate to severe renal impairment when compared to healthy subjects [13]. Type 2 diabetes puts people at greater risk for hyperlactatemia [14]. Therefore, diabetic patients who are treated with metformin have a higher tendency for the development of lactic acidosis in response to a secondary event. The cases of MALA reported in the literature were commonly encountered in ICU settings where there is an increased risk for developing acute kidney injury (AKI). 


\section{Cureus}

\section{Clinical Scenario}

Metformin-unrelated lactic acidosis

Metformin-induced lactic acidosis

Metformin-associated lactic acidosis
Features

Metformin is not detectable in the blood

Causal factors other than marked metformin accumulation are absent

Metformin detected in blood and other disease conditions are present

TABLE 2: Clinical scenarios causing lactic acidosis in patients treated with metformin

\section{Epidemiology of AKI in the ICU}

Acute kidney injury is defined as the deterioration of renal function over hours, days to weeks. The mortality rate of AKI is 50\%-80\% in intensive care unit patients [15]. AKI is an independent risk factor for increased mortality and morbidity [15]. A prospective multicenter ICU study found that patients with sepsis and AKI had a higher mortality rate ( $74 \%$ vs $45 \%$, p<0.001) than those without sepsis [16]. AKI may be community acquired or hospital acquired. Hospitalacquired AKI is associated with a worse prognosis and is often iatrogenic in nature [17]. Amongst various high-risk settings that put patients at an increased risk of AKI, infections, sepsis, shock, need for mechanical ventilation and surgery are a few worth mentioning. In 2012, the Kidney Disease Improving Global Outcomes (KDIGO) Clinical Practice Guideline for AKI consolidated the Risk Injury Failure Loss ESRD (RIFLE) criteria and Acute Kidney Injury Network (AKIN) criteria into the most recent definition and classification system for AKI as shown in Table 3 [18]. The current definition and classification of AKI rely upon functional criteria including changes in serum creatinine and urine output. More than half of the patients in the ICU develop stage 1 AKI during the ICU course. The exact incidence of developing stage 2 and $3 \mathrm{AKI}$ is not well known but is believed to be far less. And lastly, the requirement for renal replacement therapy is about 10\% [15]. Amongst the various risk factors, age, anemia, liver failure, chronic kidney disease, presence of heart failure and exposures to nephrotoxic agents are notable [19].

\begin{tabular}{|c|c|c|}
\hline & Creatinine criteria & Urine output criteria \\
\hline Risk or Stage 1 & $\begin{array}{l}\text { Creat }>0.3 \mathrm{mg} / \mathrm{dl}<48 \mathrm{hr} \text { or Creat }>150 \% \text { and }<200<7 \\
\text { d }\end{array}$ & $\mathrm{U} / \mathrm{O}<0.5 \mathrm{ml} / \mathrm{kg} / \mathrm{h}$ for $6 \mathrm{~h}$ \\
\hline Injury or Stage 2 & Creat $>200 \%$ and $<300 \%<7$ d & $\mathrm{U} / \mathrm{O}<0.5 \mathrm{ml} / \mathrm{kg} / \mathrm{h}$ for $12 \mathrm{~h}$ \\
\hline $\begin{array}{l}\text { Failure or Stage } \\
3\end{array}$ & Creat $>300 \%$, or $>4 \mathrm{mg} / \mathrm{dl}<7 \mathrm{~d}$ & $\begin{array}{l}\mathrm{U} / \mathrm{O}<0.3 \mathrm{ml} / \mathrm{kg} / \mathrm{h} \text { for } 24 \mathrm{~h} \text { or anuria for } 12 \\
\mathrm{~h}\end{array}$ \\
\hline
\end{tabular}

TABLE 3: Kidney Disease Improving Global Outcomes (KDIGO) acute kidney injury (AKI) classification

\section{Incidence of MALA}

The estimated incidence of MALA is 0.03 to 0.06 per 1000 patient-years [20]. Thus, it is very 


\section{Cureus}

rare. Literature search clearly shows that the incidence of MALA is very low and even the few cases that have been reported were associated with conditions that would predispose to lactic acidosis.

A comparative outcomes study (The COSMIC Approach Study) that looked at patients with type-2 diabetes on metformin vs. patients on non-metformin anti-hyperglycemic therapies for 1 year, found no cases of lactic acidosis. They also reported that the incidence of other serious adverse events were similar between the two groups [21]. Another meta-analysis that included 347 comparative trials and cohort studies compared patients treated with metformin with those not on metformin and concluded no significant difference in the plasma lactate levels and there were no cases of lactic acidosis [22]. In a U.K. nested case-control analysis of 50,048 patients, using a U.K-based General Practice Research Database it was revealed that there was no difference in the incidence of lactic acidosis between metformin and sulfonylurea users [23]. Of note, lactic acidosis reported in this study occurred in patients with preexisting conditions. These studies excluded the patients with renal failure (serum creatinine more than $1.4 \mathrm{mg} / \mathrm{dl}$ ). Clinical trials and case-control studies generally don't include patients at risk for MALA and hence they don't reflect the actual prevalence in the community. It is also important to understand that the true incidence of MALA is difficult to predict as there are inconsistencies in reporting metformin levels, creatinine levels, lactate levels and associated conditions [24].

\section{Mechanism of MALA}

Lactate gets oxidized to carbon dioxide and water by mitochondria to generate ATP or gets converted back to glucose by gluconeogenesis in the liver and kidney [8]. The liver can clear lactate at a very high rate and this far exceeds lactate production [7]. Therefore, lactic acidosis is rare just from increased peripheral lactate production. But, in the presence of impaired hepatic metabolism, as in liver cirrhosis, sepsis or hypoperfusion this can lead to clinically significant lactic acidosis. There are mainly two types of lactic acidosis as mentioned by Cohen and Woods. Type A usually occurs in a setting of poor tissue perfusion or oxygenation of blood and type B occurs when there is increased lactate production or reduced clearance as listed in Table $4[25]$.

\begin{tabular}{|c|c|c|}
\hline Tyре & Subtype & Conditions \\
\hline \multirow{3}{*}{$\begin{array}{l}\text { Type } A \text { (Tissue } \\
\text { hypoxia) }\end{array}$} & Systemic hypoperfusion & Shock (hypovolemic, septic, cardiogenic) \\
\hline & Local hypoperfusion & Torsion/volvulus, arterial embolism \\
\hline & $\begin{array}{l}\text { Reduced arterial oxygen } \\
\text { content }\end{array}$ & Hypoxemia, severe anemia, carbon monoxide toxicity \\
\hline \multirow{3}{*}{$\begin{array}{l}\text { Type B (No tissue } \\
\text { hypoxia) }\end{array}$} & B1- Underlying disease & Severe liver disease, malignancy, thiamine deficiency, renal failure \\
\hline & B2- Drugs/ Toxins & $\begin{array}{l}\text { Biguanides, alcohol, cyanide, acetaminophen, ethylene glycol, } \\
\text { salicylates, isoniazid, zidovudine }\end{array}$ \\
\hline & $\begin{array}{l}\text { Type B3- Congenital } \\
\text { metabolic defects }\end{array}$ & Mitochondrial disorders \\
\hline
\end{tabular}

TABLE 4: Lactic acidosis types 


\section{Cureus}

Metformin increases plasma lactate levels by inhibiting mitochondrial oxidative phosphorylation in tissues responsible for lactate removal. Metformin also inhibits hepatic gluconeogenesis, resulting in additional lactate production. This is explained in Figure 2 [8].

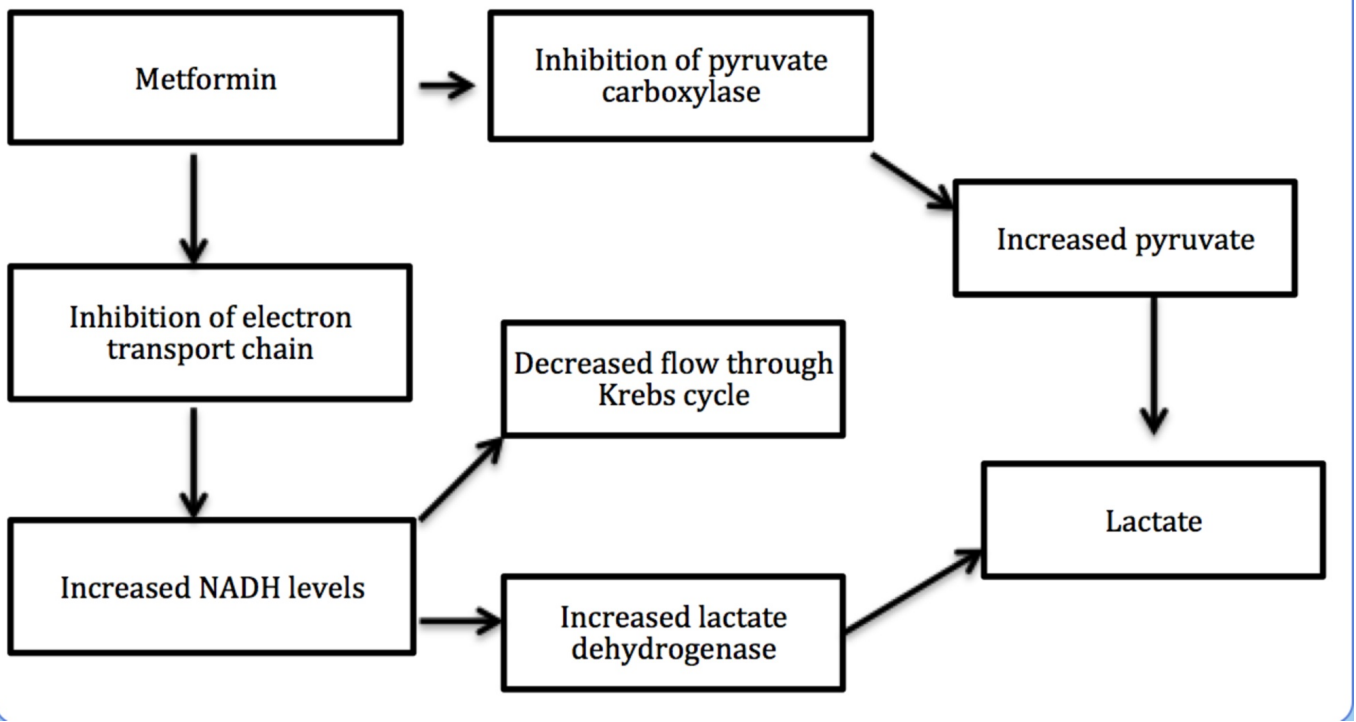

FIGURE 2: Mechanism of metformin-associated lactic acidosis (MALA)

A. Metformin inhibits pyruvate carboxylase, which results in inhibition of hepatic gluconeogenesis and in turn leads to lactate accumulation. B. Metformin also inhibits complex I of the mitochondrial electron transport chain, which increases the nicotinamide adenine dinucleotide (NADH) level, that in turn reduces the flow through the Krebs cycle and also increases lactate dehydrogenase (LDH) activity resulting in lactate accumulation.

\section{Metformin and cardiovascular diseases}

In cardiovascular ICU we routinely see patients with diabetes who are on metformin. Metformin should be continued in patients with stable coronary artery disease, acute coronary syndrome, and $\mathrm{CHF}$ as it is associated with a better cardiovascular prognosis compared to other oral glucose-lowering agents. However, this does not apply to patients in acute circulatory failure due to the potential risk of tissue hypo-perfusion [26].

\section{Metformin in patients with renal insufficiency}

Metformin is excreted through the kidneys. Therefore, its dosage should be reduced in patients with renal insufficiency in proportion to the reduced eGFR. In chronic kidney disease, the elimination of metformin is prolonged and is inversely proportional to the creatinine clearance. This explains the risk of metformin accumulation in case of renal insufficiency [27].

Chronic kidney disease (CKD) is defined as the creatinine clearance of less than $60 \mathrm{~mL} / \mathrm{min}$. In patients with type-2 diabetes mellitus and CKD, initiation of metformin is a

contraindication. Because of this contraindication, there is very limited data available regarding metformin use and the risk of lactic acidosis in patients with CKD. According to the Reduction of Atherothrombosis for Continued Health (REACH) Registry, which included a study sample of 19,691 type 2 diabetic patients with established atherothrombosis, metformin was prescribed in 1,572 patients with moderate renal failure (KDOQI stage 3 ) in contraindication to guidelines for 
its use. The results showed that when compared to other oral hypoglycemia agents, metformin use was associated with a significantly lower two-year mortality rate in patients with an eGFR of 30 to $60 \mathrm{~mL} / \mathrm{min} / 1.73 \mathrm{~m} 2$ and it was much greater than that observed in patients with normal renal function [28].

These results were further strengthened by similar findings observed in a large populationbased observational study from the Swedish National Diabetes Register which looked at 51,675 patients with type 2 diabetes and different levels of renal function [29].

In this subgroup, particular attention should be paid to the renal function prior to initiation of metformin therapy and periodically thereafter using eGFR. Assess the benefits/risks of metformin use in patients with eGFR 30 to $45 \mathrm{~mL} /$ minute/1.73 m2; if used, dosage reduction is recommended [5].

A systematic review published in 2017, looked at patients with type 2 diabetes and moderate to severe CKD and analyzed the outcomes. All studies compared metformin-based treatments to non-metformin-based regimen. On meta-analysis, the relative chance of dying during followup was $22 \%$ lower for patients taking metformin than for those not taking metformin [30].

In April 2016, the FDA revised its safety warning regarding metformin use in patients with CKD, switching from a serum creatinine-based definition for renal impairment to more inclusive criteria based on estimated glomerular filtration rate (eGFR). With this change, an estimated one million additional patients with moderate CKD (eGFR 30-60 mL/min/1.73m2) became eligible for metformin use, though severe CKD $($ eGFR $<30 \mathrm{~mL} / \mathrm{min} / 1.73 \mathrm{~m} 2)$ still remains a contraindication. With the growing population in the moderate CKD group using the metformin, closer attention should be paid for any increase in adverse events [31].

\section{Precautions for patients on metformin undergoing iodinated contrast imaging procedures in the ICU}

AKI secondary to iodinated contrast is common in critically ill patients, with a reported incidence of $10 \%$ to $20 \%$, with approximately $6 \%$ of those requiring renal replacement therapy [32]. The overall incidence today is decreasing because of various reasons. There has been more awareness, better preventive strategies, and availability of less toxic agents. Typically, AKI secondary to iodinated contrast is seen within three days and peaks in 5 days [18]. A small study evaluated 75 ICU patients with a normal baseline serum creatinine who were exposed to CT scans with an intravenous low osmolar contrast agent. The study found an increase in serum creatinine of more than $25 \%$ in $18 \%$ of the patients. On the other hand, patients who underwent CT scans and did not receive any contrast medium had no change in the serum creatinine [33].

According to the manufacturer, it is recommended to temporarily discontinue metformin at the time of or before iodinated contrast imaging procedures in patients with an eGFR 30 to 60 $\mathrm{mL} / \mathrm{minute} / 1.73 \mathrm{~m} 2$. This also applies to patients with a history of hepatic disease, alcoholism and heart failure. Patients who will receive intra-arterial iodinated contrast fall in this category as well. Reevaluation of eGFR 48 hours after imaging procedure is recommended and metformin can be restarted if the renal function is stable. The data regarding the use of iodinated contrast in patients who are on metformin is very scarce. A U.K. based study done in 1998 looked at 33 hospitalized patients who were on metformin and received iodinated contrast. Of these patients, 29 had a normal serum creatinine prior to the procedure and none of them had a rise following the procedure. The remaining four patients who had an abnormal serum creatinine prior to the procedure demonstrated a considerable deterioration and subsequently died. Two of these deaths were from unrelated causes and two were due to the 
development of acute renal failure [34].

On the other hand, the American College of Radiology (ACR) guidelines recommend that metformin may be used prior to or following administration of iodinated contrast media in patients who have no evidence of AKI and with an eGFR $\geqslant 30 \mathrm{~mL} /$ minute $/ 1.73 \mathrm{~m} 2$. They do suggest temporary discontinuation of metformin in patients with known AKI or severe chronic kidney disease ([stage IV or V [ie, eGFR $<30 \mathrm{~mL} /$ minute $/ 1.73 \mathrm{~m} 2]$ ) or those who are undergoing arterial catheter studies (ACR 2017) [35].

\section{Metformin interaction with ICU drugs}

Metformin does not have any clinically important interactions with other drugs. However, there are certain medications which are cationic agents and may compete with metformin for elimination as they are eliminated by renal tubular secretion. Some of them are amiloride, digoxin, morphine, procainamide, quinidine, quinine, ranitidine, triamterene, trimethoprim, and vancomycin. Drugs such as cimetidine, furosemide, or nifedipine may increase the concentration of metformin if taken concomitantly. So, it is important to closely monitor patients who are on metformin in association with these agents for any such potential toxicity [36].

\section{Blood glucose in ICU}

Hyperglycemia, which is defined as blood glucose concentration [BG] $>110 \mathrm{mg} / \mathrm{dL}$, is very common in the ICU and occurs in more than $80 \%$ of critically ill patients [37]. Hospitalization of patients with diabetes frequently leads to hyperglycemia or hypoglycemia by interrupting the outpatient balance of medications and diet [38]. Stress hyperglycemia occurs in hospitalized patients without evidence of pre-existing diabetes and it results from the acute metabolic and hormonal changes in response to stress and injury. There are several factors that affect blood glucose concentration in ICU patients. These include history of diabetes; insulin resistance; severity of the acute illness; enteral or parenteral glucose administration; use of steroids etc [39]. It has been well established that both variability in blood glucose levels and hypoglycemia are associated with increased mortality [40]. In light of this, the current Surviving Sepsis Campaign recommends that all patients with severe sepsis who have blood glucose levels that exceed $180 \mathrm{mg} / \mathrm{dL}$ to be started on intravenous insulin therapy. The goal is usually to maintain blood glucose between approximately 144 to $180 \mathrm{mg} / \mathrm{dL}$ [41].

Stress hyperglycemia causes increased mortality in the setting of acute myocardial infarction. Similarly, in patients who are undergoing coronary artery bypass surgery, it has been shown that perioperative glycemic control correlates with postoperative risk of nosocomial infection [42]. The rate of post-op wound infection decreased with maintaining a goal blood glucose level of less than $200 \mathrm{mg} / \mathrm{dl}$ in diabetic patients undergoing cardiac surgery [43].

\section{Management of hyperglycemia in a critical care setting}

The overall goal is to minimize blood glucose variability without causing hypoglycemia. Current recommendations suggest keeping the blood sugar below $180 \mathrm{mg} / \mathrm{dL}$ and when insulin is needed to do so, to target a blood sugar of $144-180 \mathrm{mg} / \mathrm{dL}$ [44]. In the ICU patients with diabetes, who are hyperglycemic, it is recommended to start an intravenous insulin infusion [45]. This can be demanding but it helps minimize variability in blood glucose levels, which is a common occurrence in critically ill patients. If this regimen cannot be initiated, then alternatively a long-acting subcutaneous insulin with additional short-acting insulin can be utilized [38]. Insulin pumps should be discontinued in ICU due to the risk of hypoglycemia [38].

\section{Preadmission metformin use and ICU mortality}


It is very well accepted that metformin is a great hypoglycemic agent when it comes to glycemic control. But beyond that, there is limited data regarding its benefits. The most notable benefits are its proposed anti-inflammatory effects. There are numerous proposed theories that exist regarding the possible mechanism of this effect. In a review submitted by Saisho et al., it is suggested that there is improvement in chronic inflammation by improvement of metabolic parameters. It is also proposed that metformin has direct anti-inflammatory effects and the decreased incidence of hypoglycemic events leads to a decreased sympatho-adrenergic response [46].

Christiansen and colleagues conducted a population-based study and identified the cohort by using the criteria of filled metformin prescriptions 90 days prior to the admission to ICU. It was noted that the thirty-day mortality was $17.6 \%$ in patients using metformin alone, $17.9 \%$ in patients using metformin with other oral hypoglycemic agents and $25.0 \%$ in metformin nonusers. This was true for all subgroups, including those in the medical and surgical ICUs. But, interestingly it was most significant in elderly patients and in patients with well-controlled diabetes. They concluded that metformin use prior to the admission was associated with a reduction in 30-day mortality [47].

Similarly, another cohort study done in the US on elective cardiac surgery patients found that patients on metformin had fewer postoperative complications and lower in-hospital mortality (0.7\% versus $1.4 \%)$ [48].

Although there are several proposed mechanisms linking preadmission metformin use and improved critical care outcomes secondary to its anti-inflammatory and anti-thrombotic effects, further studies are required to establish clinical outcomes.

\section{Conclusions}

In all current clinical guidelines, metformin is recommended as the first line oral hypoglycemic agent for the management of type 2 diabetes mellitus. But the main limiting factor has been the fear of MALA, especially in patients with CKD. MALA has a high mortality rate and therefore remains a concern, despite the fact that it is extremely uncommon. It is important to realize that MALA happens under conditions that promote hypoperfusion or hypoxia such as sepsis, dehydration or worsening renal, or cardiac failure; in the presence of these conditions, metformin should be promptly discontinued. Further studies are needed to assess whether routine discontinuation of metformin upon ICU admission should be considered.

\section{Additional Information \\ Disclosures}

Conflicts of interest: In compliance with the ICMJE uniform disclosure form, all authors declare the following: Payment/services info: All authors have declared that no financial support was received from any organization for the submitted work. Financial relationships: All authors have declared that they have no financial relationships at present or within the previous three years with any organizations that might have an interest in the submitted work. Other relationships: All authors have declared that there are no other relationships or activities that could appear to have influenced the submitted work.

\section{References}

1. Goodarzi MO, Bryer-Ash M: Metformin revisited: re-evaluation of its properties and role in the pharmacopoeia of modern antidiabetic agents. Diabetes Obes Metab. 2005, 7:654-665. 10.1111/j.1463-1326.2004.00448.x

2. UK Prospective Diabetes Study (UKPDS) Group: Effect of intensive blood-glucose control with 
metformin on complications in overweight patients with type 2 diabetes (UKPDS 34). Lancet. 1998, 352:854-865. 10.1016/S0140-6736(98)07037-8

3. Nathan DM, Buse JB, Davidson MB, Ferrannini E, Holman RR, Sherwin R, Zinman B: Medical management of hyperglycemia in type 2 diabetes: a consensus algorithm for the initiation and adjustment of therapy: a consensus statement of the American. Diabetes Care. 2009, 32:193-203. 10.2337/dc08-9025

4. Renda F, Mura P, Finco G, Ferrazin F, Pani L, Landoni G: Metformin-associated lactic acidosis requiring hospitalization. A national 10 year survey and a systematic literature review. Eur Rev Med Pharmacol Sci. 2013, 1:45-49.

5. Graham GG, Punt J, Arora M, et al.: Clinical pharmacokinetics of metformin. Clin Pharmacokinet. 2011, 50:81-98. 10.2165/11534750-000000000-00000

6. Reinehr T, Kiess W, Kapellen T, Andler W: Insulin sensitivity among obese children and adolescents, according to degree of weight loss. Pediatrics. 2004, 114:1569-1573. 10.1542/peds.2003-0649-F

7. Fall PJ, Szerlip HM: Lactic acidosis: from sour milk to septic shock . J Intensive Care Med. 2005, 5:255-271. 10.1177/0885066605278644

8. Wang DS, Kusuhara H, Kato Y, Jonker JW, Schinkel AH, Sugiyama Y: Involvement of organic cation transporter 1 in the lactic acidosis caused by metformin. Mol Pharmacol. 2003, 63:844848. 10.1124/mol.63.4.844

9. Owen MR, Doran E, Halestrap AP: Evidence that metformin exerts its anti-diabetic effects through inhibition of complex 1 of the mitochondrial respiratory chain. Biochem J. 2000, 348:607-614. 10.1042/bj3480607

10. Lalau JD, Arnouts P, Sharif A, De Broe ME: Metformin and other antidiabetic agents in renal failure patients. Kidney Int. 2015, 87:308-322. 10.1038/ki.2014.19

11. Lalau JD, Lemaire-Hurtel AS, Lacroix C: Establishment of a database of metformin plasma concentrations and erythrocyte levels in normal and emergency situations. Clin Drug Investig. 2011, 31:435-438. 10.2165/11588310-000000000-00000

12. Misbin RI, Green L, Stadel BV, Gueriguian JL, Gubbi A, Fleming GA: Lactic acidosis in patients with diabetes treated with metformin. N Engl J Med. 1998, 338:265-266.

10.1056/NEJM199801223380415

13. Frid A, Sterner GN, Londahl M, Wiklander C, Cato A, Vinge E, Andersson A: Novel assay of metformin levels in patients with type 2 diabetes and varying levels of renal function: clinical recommendations. Diabetes Care. 2010, 33:1291-1293. 10.2337/dc09-1284

14. Guelho D, Paiva I, Carrilho F: Relation between metformin treatment and the development of hyperlactacidemia at the emergency room [Article in English, Portuguese]. Acta Med Port. 2014, 27:196-203. 10.20344/amp.4071

15. Bellomo R, Ronco C, Mehta RL, et al.: Acute kidney injury in the ICU: from injury to recovery: reports from the 5th Paris International Conference. Ann Intensive Care. 2017, 7:49.

10.1186/s13613-017-0260-y

16. Neveu H, Kleinknecht D, Brivet F, Loirat P, Landais P: Prognostic factors in acute renal failure due to sepsis. Results of a prospective multicentre study. Nephrol Dial Transplant. 1996, 11:293-299. 10.1093/oxfordjournals.ndt.a027256

17. Wonnacott A, Meran S, Amphlett B, Talabani B, Phillips A: Epidemiology and outcomes in community-acquired versus hospital-acquired AKI. Clin J Am Soc Nephrol. 2014, 6:1007-1014. 10.2215/CJN.07920713

18. KDIGO: Kidney disease: improving global outcomes (KDIGO) acute kidney injury work group . Kidney Int. 2012, 2:1. 10.1038/kisup.2012.1

19. Chawla LS, Abell L, Mazhari R, et al.: Identifying critically ill patients at high risk for developing acute renal failure: a pilot study. Kidney Int. 2005, 5:2274-2280. 10.1111/j.15231755.2005.00686.x

20. Bailey CJ, Turner RC: Metformin. N Engl J Med. 1996, 334:574-579. 10.1056/NEJM199602293340906

21. Cryer DR, Nicholas SP, Henry DH, Mills DJ, Stadel BV: Comparative outcomes study of metformin intervention versus conventional approach the COSMIC Approach Study. Diabetes Care. 2005, 28:539-543. 10.2337/diacare.28.3.539

22. Salpeter SR, Greyber E, Pasternak GA, Salpeter EE: Risk of fatal and nonfatal lactic acidosis with metformin use in type 2 diabetes mellitus. Cochrane Database Syst Rev. 2010, 4:CD002967. 10.1002/14651858.CD002967.pub4 
23. Bodmer M, Meier C, Krahenbuhl S, Jick SS, Meier CR: Metformin, sulfonylureas, or other antidiabetes drugs and the risk of lactic acidosis or hypoglycemia: a nested case-control analysis. Diabetes Care. 2008, 11:2086-2091. 10.2337/dc08-1171

24. Lalau JD, Race JM: Lactic acidosis in metformin therapy: searching for a link with metformin in reports of 'metformin-associated lactic acidosis'. Diabetes Obes Metab. 2001, 3:195-201. 10.1046/j.1463-1326.2001.00128.x

25. Cohen RD, Woods HF: Clinical and Biochemical Aspects of Lactic Acidosis. Blackwell Scientific Publications, New Jersey, USA; 1976.

26. Masoudi FA, Inzucchi SE, Wang Y, Havranek EP, Foody JM, Krumholz HM: Thiazolidinediones, metformin, and outcomes in older patients with diabetes and heart failure: an observational study. Circulation. 2005, 111:583-590. 10.1161/01.CIR.0000154542.13412.B1

27. Scheen AJ, Paquot N: Metformin revisited: a critical review of the benefit-risk balance in atrisk patients with type 2 diabetes [Article in English, French]. Diabetes Metab. 2013, 39:179190. 10.1016/j.diabet.2013.02.006

28. Roussel R, Travert F, Pasquet B, et al.: Metformin use and mortality among patients with diabetes and atherothrombosis. Arch Intern Med. 2010, 170:1892-1899.

10.1001/archinternmed.2010.409

29. Ekstrom N, Schioler L, Svensson AM, et al.: Effectiveness and safety of metformin in 51675 patients with type 2 diabetes and different levels of renal function: a cohort study from the Swedish National Diabetes Register. BMJ Open. 2012, 2:4. 10.1136/bmjopen-2012-001076

30. Crowley MJ, Diamantidis CJ, McDuffie JR, et al.: Clinical outcomes of metformin use in populations with chronic kidney disease, congestive heart failure, or chronic liver disease: a systematic review. Ann Intern Med. 2017, 166:191-200. 10.7326/M16-1901

31. FDA revises warnings regarding use of the diabetes medicine metformin in certain patients with reduced kidney function. (2016). Accessed: May 1, 2019: http://www.fda.gov/downloads/Drugs/DrugSafety/UCM494140.pdf.

32. Case J, Khan S, Khalid R, Khan A: Epidemiology of acute kidney injury in the intensive care unit. Crit Care Res Pract. 2013, 1-9. 10.1155/2013/479730

33. Polena S, Yang S, Alam R, et al.: Nephropathy in critically Ill patients without preexisting renal disease. Proc West Pharmacol Soc. 2005, 48:134-135.

34. Nawaz S, Cleveland T, Gaines PA, Chan P: Clinical risk associated with contrast angiography in metformin treated patients: a clinical review. Clin Radiol. 1998, 53:342-44.

35. ACR manual on contrast media . (2017). Accessed: May 1, 2019: https://wwwacrorg/QualitySafety/Resources/Contrast-Manual 2017.

36. Rojas LB, Gomes MB: Metformin: an old but still the best treatment for type 2 diabetes . Diabetol Metab Syndr. 2013, 5:6. 10.1186/1758-5996-5-6

37. Alter D, Deines G: Tight glycemic control and point-of-care testing. Clin Lab Med. 2009, 29:511-522. 10.1016/j.cll.2009.06.009

38. Boord JB, Graber AL, Christman JW, Powers AC: Practical management of diabetes in critically ill patients. Am J Respir Crit Care Med. 2001, 164:1763-1767. 10.1164/ajrccm.164.10.2103068

39. Hsu CW: Glycemic control in critically ill patients. World J Crit Care Med. 2012, 1:31-39. 10.5492/wjccm.v1.i1.31

40. Hermanides J, Bosman RJ, Vriesendorp TM, et al.: Hypoglycemia is associated with intensive care unit mortality. Crit Care Med. 2010, 38:1430-1434. 10.1097/CCM.0b013e3181de562c

41. Rhodes A, Evans LE, Alhazzani W, et al.: Surviving sepsis campaign: International guidelines for management of sepsis and septic shock. Intensive Care Med. 2017, 43:304-377.

10.1007/s00134-017-4683-6

42. Golden SH, Peart-Vigilance C, Kao WH, Brancati FL: Perioperative glycemic control and the risk of infectious complications in a cohort of adults with diabetes. Diabetes Care. 1999, 22:1408-1414. 10.2337/diacare.22.9.1408

43. Furnary AP, Zerr KJ, Grunkemeier GL, Starr A: Continuous intravenous insulin infusion reduces the incidence of deep sternal wound infection in diabetic patients after cardiac surgical procedures. Ann Thorac Surg. 1999, 2:352-360.

44. NICE-SUGAR Study investigators: Intensive versus conventional glucose control in critically ill patients. N Engl J Med. 2009, 360:1283-1297. 10.1056/NEJMoa0810625

45. Hirsch IB, Paauw DS, Brunzell J: Inpatient management of adults with diabetes . Diabetes Care. 1995, 18:870-878. 10.2337/diacare.18.6.870 


\section{Cureus}

46. Saisho Y: Metformin and inflammation: its potential beyond glucose-lowering effect . Endocr Metab Immune Disord Drug Targets. 2015, 15:196-205. 10.2174/1871530315666150316124019

47. Christiansen C, Johansen M, Christensen S, O'Brien JM, Tonnesen E, Sorensen H:

Preadmission metformin use and mortality among intensive care patients with diabetes: a cohort study. Crit Care. 2013, 17:R192. 10.1186/cc12886

48. Duncan AI, Koch CG, Xu M, Manlapaz M, Batdorf B, Pitas G, Starr N: Recent metformin ingestion does not increase in-hospital morbidity or mortality after cardiac surgery. Anesth Analg. 2007, 104:42-50. 10.1213/01.ane.0000242532.42656.e7 